\title{
Clinical And Etiological Profile of Children Aged 1 Month To 60 Months Admitted with Microcephaly in Bal Chikitsalaya, Maharana Bhupal Goverment Hospital, RNT Medical College , Udaipur (Rajasthan)
}

\author{
Neeraj Kumar ${ }^{1}$, Suresh Goyal ${ }^{2}$, Bharat Lal Sharma ${ }^{3}$, Bhupesh Jain ${ }^{4}$ \\ ${ }^{1}$ Postgraduate MD Student, ${ }^{2}$ Senior Professor \& HOD, ${ }^{3}$ Postgraduate MD Student, ${ }^{4}$ Assistant Professor \\ Department Of Pediatrics, RNT Medical College, Udaipur (Raj.), India
}

\begin{abstract}
:
Objective: To study the clinical and etiological profile with the change in brain by neuroimaging (MRI brain) in children with microcephaly aged 1 month to 60 months.

Materials and Methods: Study Design - Hospital based prospective study. Children attending Bal Chikitsalaya, Maharana Bhupal Goverment Hospital, RNT Medical College, Udaipur (Rajasthan) were evaluated as per protocol. Z scores of head circumference were calculated using WHO charts and clinical and etiological profile with the change in brain by neuroimaging (MRI brain) of those with microcephaly.

Results: 103 children with microcephaly were enrolled in the study. 58 (56.31\%) were male and 45 (43.68\%) were female. Male to female ratio was 1.29:1. Mean age of children with microcephaly was 19.71 \pm 14.79 months. Most children with microcephaly presented with developmental delay 93(90.29\%), abnormal tone 75(72.82\%), convulsion 29(28.15\%), regression of mile stone 13(12.62\%), tremor 11(10.68\%), visual abnormalities 12(11.65\%), hearing abnormalities 10(9.71\%) and dysmorphic facies 9(8.74\%). The patients were classified according to the final diagnosis into 3 groups; group I: primary microcephaly $-12(11.65 \%)$ cases, group II: secondary microcephaly - 88(85.44\%) cases and group III: undiagnosed - 3(2.91\%). The most frequent MRI findings were cerebral atrophy in 50(48.54\%) cases followed by changes suggestive of hypoxic ischemic encephalopathy (HIE) 19(18.94\%) cases.

Conclusion: Among the microcephaly children admitted in hospital, severe acute malnutrition was the commonest cause followed by cerebral palsy. Cerebral atrophy was most frequent finding on neuroimaging (MRI) followed by changes suggestive of hypoxic ischemic encephalopathy.
\end{abstract}

Keywords: Cerebral atrophy, Cerebral palsy, Developmental delay, Microcephaly, Neuroimaging

\section{Introduction}

Microcephaly is a descriptive term that refers to a cranium that is significantly smaller than the standard for the individual ${ }^{\mathrm{s}}$ age and sex. It should usually be considered as a neurologic sign and defined as "Occipitofrontal head circumference (OFC) more than 3 SDs below the mean for age and gender due to small brain $[4,7,15]$. Microcephaly may be described as primary and secondary. Primary microcephaly in which the brain fails to grow to the correct size during pregnancy[3]. Secondary (also known as acquired) microcephaly is a condition in which a child's head circumference is within the normal range at birth and for an undefined period thereafter, but then does not increase as fast as normal and result in small head(microcephaly) [5]. Comprehensive study on microcephaly is not available in the literature. Hence the present study has been planned with aims to study the clinical and etiological profile of children with microcephaly and to evaluate change in brain by neuroimaging (MRI).

\section{Materials and Methods}

103 children with microcephaly were enrolled in the study. The study was conducted on all the children aged 1 month to 60 months with microcephaly (HC<-3SD) admitted in Bal Chikitsalaya, Maharana Bhupal Government Hospital, RNT medical college, Udaipur, Rajasthan, India during a period of 12 months from January 2015 to December 2015. Approval was taken from the hospital ethical committee. A written informed consent was taken from the parents.

Inclusion Criteria:

1. Children aged 1 month to 60 months.

2. Head circumference <-3SD.

Exclusion Criteria:

1. Children aged $<1$ month and $>60$ months.

2. Head circumference $>-3 \mathrm{SD}$. 
The head circumference was measured by placing a non-stretchable tape around the cranial vault to include the widest part of the forehead and the most prominent part of the occipital area to arrive at the largest possible measurement [3]. Microcephaly was defined as head circumference (HC) less than 3 SD or Z score < 3. WHO growth charts were used for assessing anthropometric parameters [1]. Detailed history (antenatal, natal, postnatal, developmental and family), general and systemic examination was carried out. Screening of developmental delay by Denver developmental screening-II [6] and Gesell`s developmental schedule [10].

\section{Investigations:}

Test done in all children: Neuroimaging (MRI Brain) - MRI brain was done in all children included in the study. The head coil was used for the scan. It was performed on 1.5 Tesla unit (Philips achiva machine) using a head coil. Cerebral atrophy was defined by bifrontal index [12], bicaudate index [8] and width of sylvian fissure [13].

Blood samples: CBC, blood sugar, serum urea, serum creatine, serum electrolyte and LFT.

Urine examination: routine, urine ketone and reducing substrate.

Fundus

Other Test (If indicated and possible):

Blood samples: (Serum ammonia, serum lactate, arterial blood gases thyroid profile and TORCH), Genetic testing - (e.g. - karyotyping, subtelomeric fluorescent in situ hybridization), Metabolic testing - (e.g.-Tandem mass spectrometry (TMS) and organic acids profile in urine using Gas chromatography mass spectrometry), EEG and Screening for ophthalmological (e.g. visual evoked potential) and audiological abnormalities (e.g.BERA and audiometry, structural anomalies and malformation).

All the collected data was managed and analysed with standard software of Biostatics (SPSS Version 20).

\section{Results}

103 children with microcephaly were enrolled in the study. $58(56.31 \%)$ were male and $45(43.68 \%)$ were female. Male to female ratio was $1.29: 1$. Mean age of children with microcephaly was $19.71 \pm 14.79$ months. Nutrition status of microcephaly children was recorded using weight for height criteria. 65(63.11\%) had SAM and 31(30.10\%) had moderate acute malnutrition. Among children with microcephaly, birth asphyxia $15(14.56 \%$ ) and intra uterine growth retardation (IUGR) $15(14.56 \%)$ were two most common adverse perinatal events followed by neonatal seizure $9(8.74 \%)$, preterm $9(8.74 \%)$, neonatal jaundice $4(3.88 \%)$ and neonatal infection $2(1.94 \%)$. Anaemia $(\mathrm{Hb}<11 \mathrm{~g} / \mathrm{dl})$ was present in $82(79.62 \%)$ children of microcephaly.

Most of children with microcephaly presented with developmental delay 93(90.29\%), abnormal tone $75(72.82 \%)$ and convulsion 29(28.15\%) [generalised $24(23.30 \%) \&$ focal 5(4.85\%)]. Other important clinical features were cerebral palsy $21(20.39 \%)$, regression of mile stone 13(12.62\%), tremor 11(10.68\%), visual abnormalities $12(11.65 \%)$, hearing abnormalities $10(9.71 \%)$, dysmorphic facies $9(8.74 \%)$, infantile spasm $2(1.94 \%)$ and chorioathetoid type movement 2(1.94\%). Among children with microcephaly Developmental Quotient (DQ) $\leq 70 \%$ was present in $87(87.88 \%$ ) children out of 99 . Mean DQ of children with microcephaly was $48.74 \pm 18.45$. Among the cerebral palsy spastic quadriplegia $\mathrm{CP}$ was seen in $11(52.38 \%)$ children followed by and spastic diplegia CP 5(23.81\%) children, hemiplegic CP 3(14.29\%) children and choreoathetoid CP $2(9.52 \%)$ children. Among the cerebral palsy spastic CP was seen in $19(90.48 \%)$ children followed by hypotonic CP 2(9.52\%).

Table (1) demonstrates the etiological profile of the children with microcephaly. Etiology of microcephaly classified according to the final diagnosis into 3 groups; group I: primary microcephaly $12(11.65 \%)$ cases, group II: secondary microcephaly - 88(85.44\%) cases and group III: undiagnosed $3(2.91 \%)$. No child was diagnosed as familial microcephaly. Secondary microcephaly was directly due to SAM seen in 53(51.46\%) and association with other causes was seen in 12 cases (12.98\%) - SAM with ITS 5(4.85\%), SAM with HIE 4(3.88\%), SAM with Down syndrome 1(0.97\%), SAM with Goldenhar syndrome $1(0.97 \%)$ \& SAM with VACTERL association $1(0.97 \%)$. Secondary microcephaly due to cerebral palsy was seen in $21(20.39 \%)$ children. Cerebral palsy was most common due to perinatal asphyxia - 14(66.67\%) children followed by preterm - 4(19.05\%) children, hyperbilirubinemia encephalopathy (Kernicterus) - 1(4.76\%) children, intra cranial hemorrhage (ICH) - 1(4.76\%) children and benign enlargement of subarachnoid space 1(4.76\%). Among the Hypoxic Ischemic Encephalopathy was most common due to perinatal asphyxia 14(73.68\%) children followed by preterm - 4(21.05) children. One Down syndromic children had Hypoxic Ischemic Encephalopathy in MRI brain. Other causes of secondary microcephaly were seen in 7 cases (ITS 6(5.83\%), TORCH 2(1.94\%) \& CHD 1(0.97\%). 
Clinical And Etiological Profile Of Children Aged Month To 60 Months...

Table (1):- Etiological profile of children with Microcephaly

\begin{tabular}{|l|l|l|l|}
\hline & \multicolumn{1}{|c|}{ Final Diagnosis } & No. of children (\%) \\
\hline $\begin{array}{l}\text { (A) Primary Microcephaly } \\
12(11.65 \%)\end{array}$ & $\begin{array}{l}\text { i. Syndromic } \\
9(8.74 \%) \\
\end{array}$ & & Syndrome with SAM(1)
\end{tabular}

Table (2) demonstrates the change in brain by neuroimaging (MRI brain) in children with microcephaly. MRI brain was done in all the children enrolled in the study. Neuroimaging (MRI) was found abnormal in $81(78.64 \%)$ children. Neuroimaging was normal in 22(21.36\%) children. The most frequent MRI findings were cerebral atrophy in $50(48.54 \%)$ cases followed by changes suggestive of Hypoxic Ischemic Encephalopathy (HIE) 19(18.94\%) cases. Among the Hypoxic Ischemic Encephalopathy periventricular leucomalacia was seen in 12(63.16\%) cases. Finding of TBME with cerebral atrophy (dilatation of ventricle, periventricular CSF oozing, tuberculomas, increased vascularity \& post contrast meningeal enhancement) was seen in $5(4.85 \%)$ cases. Hypomyelination and/or delayed myelination were seen in 2(1.94\%) cases. Malformation of brain was seen in 2(1.94\%) cases (Agyria - pachygyria complex with partial agenesis /hypoplastic corpus callosum and colpocephaly - 1 case \& Vermian - Cerebellar agenesis 1 case). Organised haemorragic lesion with cystic leukomalacia was seen in $1(0.97 \%)$ case. Benign enlargement of subarachnoid space was seen in $1(0.97 \%)$ case. Symmetrical abnormal signal in b/l globus pallidus with cereral atrophy was seen in $1(0.97 \%)$ case. Among the SAM children with microcephaly cerebral atrophy (dilated ventricle, prominent sylvian fissures) was most common finding seen in $42(64.62 \%)$ children.

Table (2):- The change in brain by Neuroimaging (MRI) in children with Microcephaly

\begin{tabular}{|c|c|c|c|}
\hline S. No. & \multicolumn{2}{|r|}{ Neuroimaging (MRI) finding } & No. of children (\%) \\
\hline 1 & \multicolumn{2}{|l|}{ Cerebral Atrophy } & $50(48.54 \%)$ \\
\hline 2 & \multicolumn{2}{|l|}{ Cerebral Atrophy with TBME } & $5(4.85 \%)$ \\
\hline \multirow[t]{7}{*}{3} & \multicolumn{2}{|c|}{$\begin{array}{l}\text { Hypoxic } \quad \text { Ischemic ii. Leukomalacia } \\
\text { Encephalopathy } 19(18.94 \%)\end{array}$} & $4(3.88 \%)$ \\
\hline & & i. Leukomalacia with cerebral atrophy & $8(7.77 \%)$ \\
\hline & & iii. Leukomalacia with delay myelination & $1(0.97 \%)$ \\
\hline & & iv. Leukomalacia with gliosis in right hippocampus & $1(0.97 \%)$ \\
\hline & & $\begin{array}{l}\text { iii. abnormal signal in the Basal ganglia, thalami, } \\
\text { internal capsule, frontal \& parietooccipetal cortex } \\
\text { lesion suggestive of chronic hypoxic ischemic injury }\end{array}$ & $3(4.61 \%)$ \\
\hline & & $\begin{array}{l}\text { iv. Area of gliosis in } \mathrm{B} / \mathrm{L} \text { high parietal region with } \\
\text { thinning of posterior half of body of carpus callosum }\end{array}$ & $1(0.97 \%)$ \\
\hline & & $\begin{array}{l}\text { v. Symmetrical area of abnormal signal in the } \mathrm{B} / \mathrm{L} \\
\text { thalami, periventricular \& deep subcortical white } \\
\text { matter, predominantly in the } \mathrm{B} / \mathrm{L} \text { frontal lobe along the }\end{array}$ & $1(0.97 \%)$ \\
\hline
\end{tabular}


Clinical And Etiological Profile Of Children Aged Month To 60 Months...

\begin{tabular}{|l|l|l|}
\hline & \multicolumn{1}{|c|}{$\begin{array}{l}\text { rolantic fissure with loss of volume of white matter } \\
\text { (cerebral atrophy) }\end{array}$} & \\
\hline 4 & $\begin{array}{l}\text { Malformation of } \\
2(1.94 \%)\end{array} \quad \begin{array}{c}\text { braini. Agyria - pachygyria complex with partial agenesis } \\
\text { /hypoplastic corpus callosum and colpocephaly }\end{array}$ & $(0.97 \%)$ \\
\hline & \multicolumn{1}{|c|}{ ii. Vermian - Cerebellar agenesis } & $1(0.97 \%)$ \\
\hline 5 & Hypomyelination and/or Delayed Myelination & $2(1.94 \%)$ \\
\hline 6 & Organised Haemorragic Lesion With Cystic Leukomalacia & $1(0.97 \%)$ \\
\hline 7 & Benign enlargement of subarachnoid space & $1(0.97 \%)$ \\
\hline 8 & Symmetrical abnormal signal in b/l globus pallidus with cereral atrophy & $1(0.97 \%)$ \\
\hline 7 & Normal & $22(21.36 \%)$ \\
\hline & Total & 103 \\
\hline
\end{tabular}

Some important Neuroimage of children with Microcephaly:

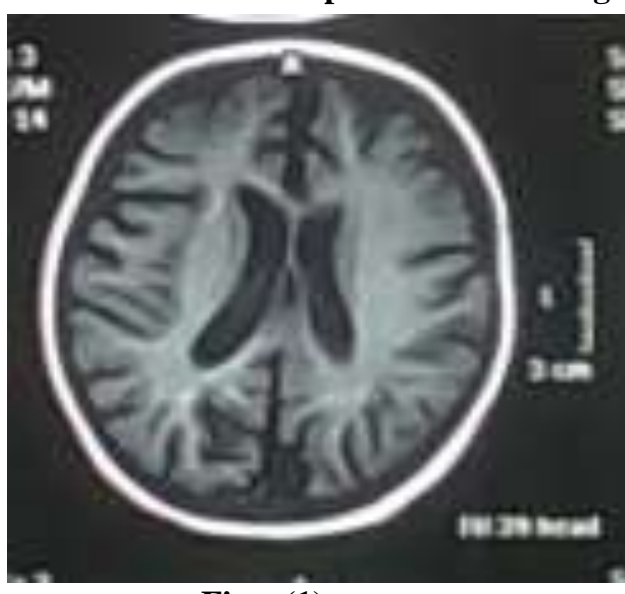

Fig.- (1)

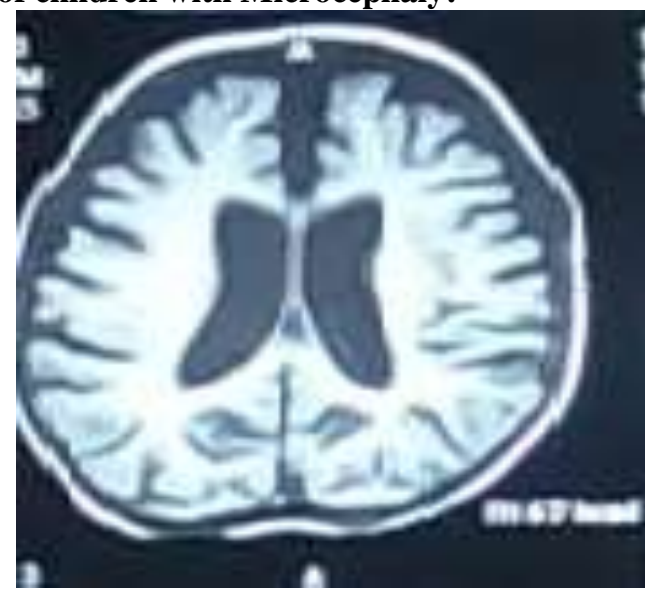

Fig. - (2)

Fig. - (1) \& (2) T1 weighted MRI images shows diffuse cerebral atrophy in the form of dilatation of ventricular system and prominence of cortical sulci \& fissures with loss of volume of white matter.

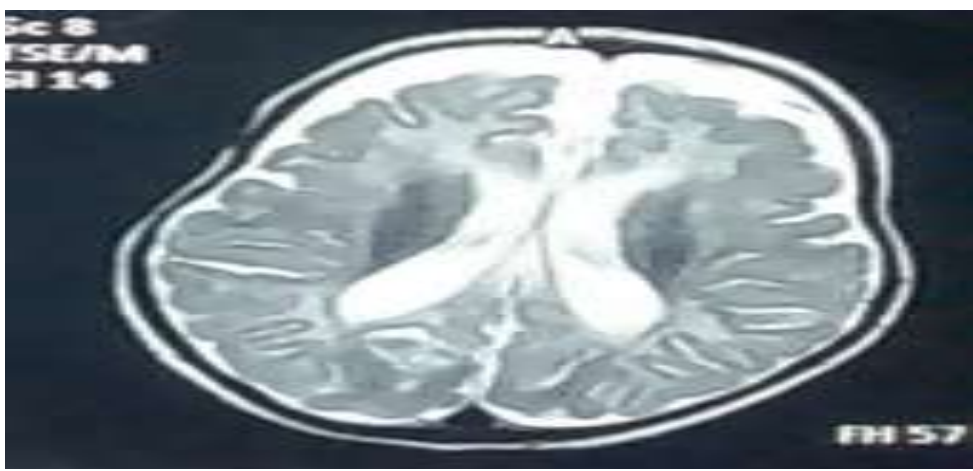

Fig. - (3)

Fig. - (3) T2 weighted MRI image shows diffuse cerebral atrophy in the form of dilatation of ventricular system and prominence of cortical sulci \& fissures with delayed myelination

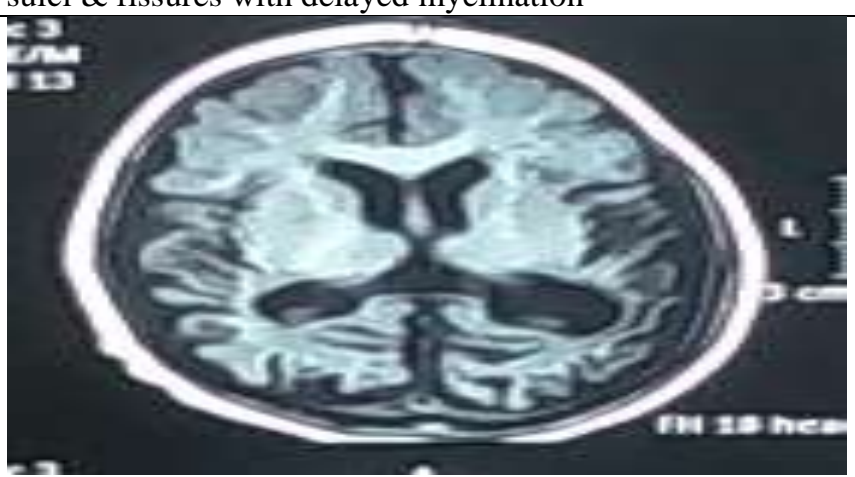

Fig. - (4) 
Fig. - (4) T1 weighted MRI image shows multiseptate cystic lesions in periventricular and deep white matter of bilateral temporal, parietal and occipital lobes appears hypointense with thinning of the cortical gyri and crowding of sulci with loss of volume of white matter and ex - vacuo dilatation of lateral ventrical, suggestive of cystic leukomalacia.

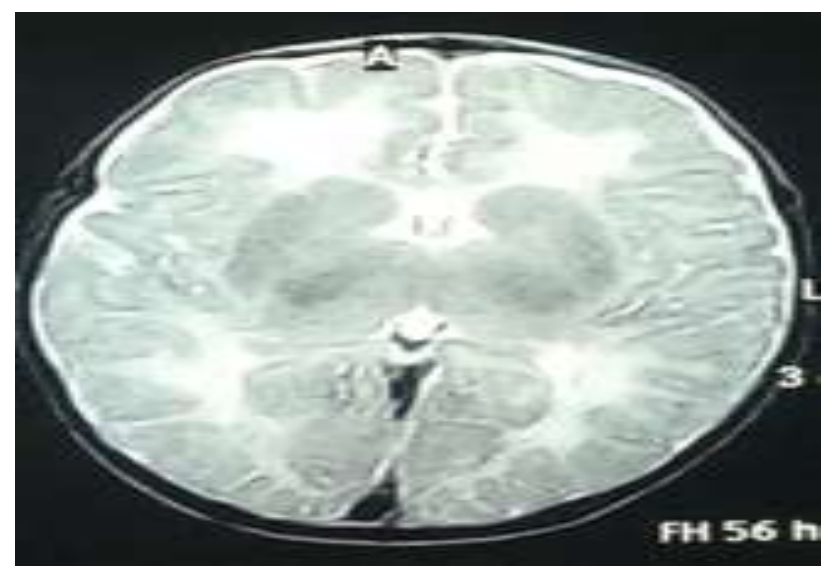

Fig. - (5)

Fig. - (5) T2 weighed MRI image shows symmetrical abnormal signals in the periventricular white matter of frontal \& parietal lobes appears hyperintense, suggestive of periventricular leukomalacia.

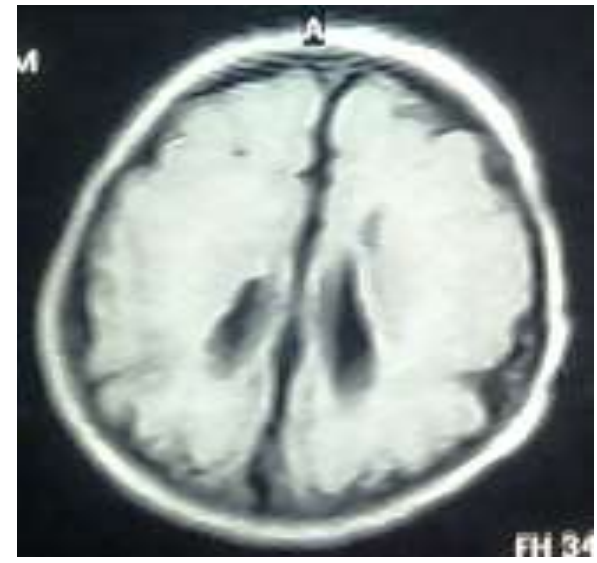

Fig. $-(6)$

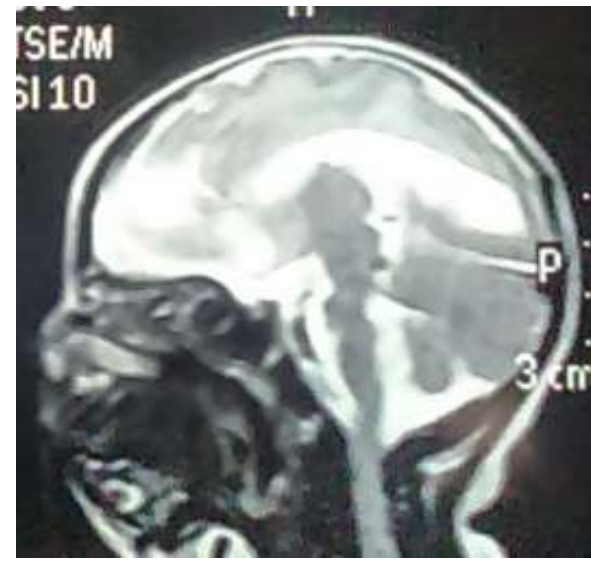

Fig. - (7)

Fig. - (6) T1 weighted MRI image shows paucity of cortical sulci with poor gray white matter interface and smooth appearing of brain parenchyma, likely representing agyria - pachygyria complex. Fig. - (7) T2 weighted MRI image shows thinning of body and splenium of corpus callosum with dilated occipital horn of lateral ventrical. MRI finding of fig. (6) \& (7) are suggestive of agyria - pachygyria complex with partial agenesis / hypoplastic corpus callosum.

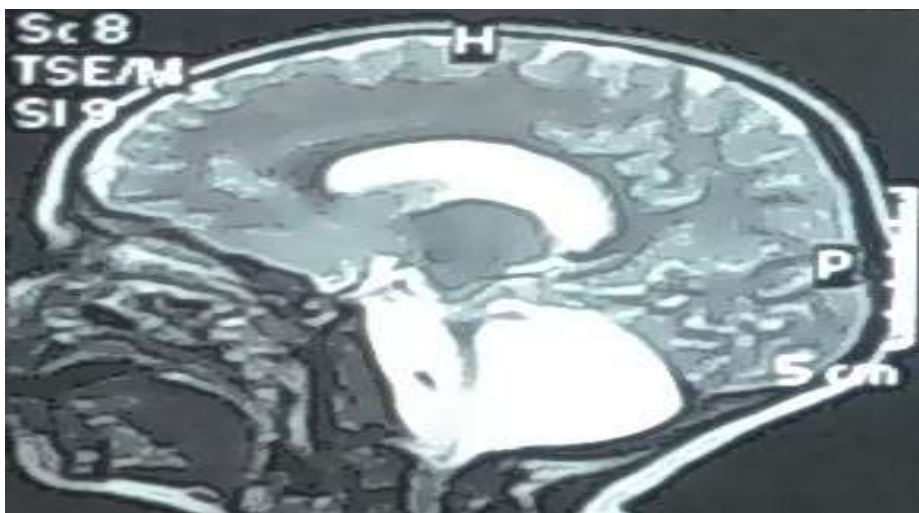

Fig. - (8) 
Fig. - (8) T2 weighted MRI image shows CSF signal intensity cystic lesion occupying almost entire posterior fossa with non - visualization of cerebellar hemisphere including vermis only small residual vermian part is seen its superior aspect with thinned out / atrophied middle and superior peduncles, these findings are suggestive of vermian - cerebellar agenesis.

\section{Discussion}

A total of 103 children with microcephaly were enrolled during study period. $58(56.31 \%)$ children were male and 45(43.68\%) children were female. Male to Female ratio was 1.29:1. Mean age of children with microcephaly was $19.71 \pm 14.79$ months. Higher prevalence of microcephaly among male children have been observed in other studies[2,9,11]. In present study most of the children 60(58.25\%) were admitted because of not growing well/ loss of weight/ not eating well and had severe acute malnutrition (SAM). Microcephaly was an incidental finding. Among children with microcephaly, 65(63.11\%) children had SAM. Higher prevalence of severe degree of malnutrition in children with microcephaly has been reported in other study [11]. Among children with microcephaly, birth asphyxia 15(14.56\%) and intra uterine growth retardation (IUGR) $15(14.56 \%$ ) were two most common adverse perinatal events followed by neonatal seizure $9(8.74 \%)$, preterm $9(8.74 \%)$, neonatal jaundice $4(3.88 \%)$ and neonatal infection $2(1.94 \%)$.

Study from India in April 2007 - April 2009 [2] on clinical profile of children with developmental delay and microcephaly has been reported significant maternal history in $14(6.06 \%)$ children, prematurity $43(18.61 \%)$, birth asphyxia $121(52.38 \%)$ and neonatal jaundice $46(19.91 \%)$ children with microcephaly (HC <3SD). In present study most of children with microcephaly were present with developmental delay 93(90.29\%). Elham et al. [9] has been reported developmental delay in 55(100\%) children with microcephaly.

In present study abnormal tone was present in $75(72.82 \%)$. Similar findings have been observed in other study[14]. In present study convulsion was present in $29(28.15 \%)$ children. Anju et al. has been reported convulsion in $198(85.71 \%)$ children with microcephaly $(\mathrm{HC}<-3 \mathrm{SD})$. In present study among children with microcephaly cerebral palsy was present in 21(20.39\%) children. Among the cerebral palsy spastic quadriplegia $\mathrm{CP}$ was seen in $11(52.38 \%)$ children followed by spastic diplegia CP $5(23.81 \%)$ children, hemiplegic CP $3(14.29 \%)$ children and choreoathetoid CP 2(9.52\%) children. Among the cerebral palsy spastic CP was seen in $19(90.48 \%)$ children followed by hypotonic CP $2(9.52 \%)$. Anju et al. has been reported cerebral palsy in 160(69.26\%) children with microcephaly (HC < - 3SD) and spastic cerebral palsy was most common (Spastic $95.7 \%$, hypotonic $3.1 \%$, dystonic $1.2 \%$ ).

In present study visual and hearing abnormalities were present in $12(11.65 \%)$ and $10(9.71 \%)$ children respectively. Anju et al. has been reported visual abnormalities 61(26.41\%) children and hearing abnormalities $39(16.88 \%)$ children with microcephaly (HC < - 3SD). In present study among children with microcephaly Developmental Quotient (DQ) $\leq 70 \%$ was present in $87(87.88 \%$ ) children out of 99 . Mean DQ of children with microcephaly was 48.74 \pm 18.45 . Aggarwal et al. has been reported DQ (mean, SD) $-29.75 \pm 17.8$.

In present study microcephaly was most commonly seen in SAM followed by cerebral palsy. Elham et al. has been reported Hypoxic ischemic encephalopathy most common causes followed by Neurometabolic causes. In present study was different from Elham A et al. because Elham A et al. was not consider nutrition status of children with microcephaly and study conducted at general pediatric and neurology clinic of Benha University Hospital (BUH) Benha, Egypt and clinic of inherited metabolic disorder at the centre of social and preventive medicine of Cairo University Children Hospital (CUCH) Cairo, Egypt. In present study conducted in general pediatric hospital. Study from India in 1995 by Prasad et al. [11] on etiology of 82 microcephalic children with developmental delay concluded definite etiology in $56 \%$, genetic causes being most common. Anju et al. [2] has been reported that syndromic primary microcephaly was seen in 13 (Down syndrome-11, rubystien taybi-1, Sjorgen-Larsson syndrome-1).

Secondary microcephaly was due to cerebral palsy associated with perinatal hypoxia and periventricular leucomalacia was seen in $69.3 \%$ of children. In present study was different from Prasad et al. and Aggarwal et al. because in both the studies nutrition status of children with microcephaly was not assessed.

In present study Neuroimaging (MRI) was found abnormal in $81(78.64 \%)$ children. Normal brain MRI was seen in $22(21.36 \%)$ children. The yield of neuroimaging same was reported by other studies [2,9]. In present study the most frequent MRI findings were cerebral atrophy in 50(48.54\%) cases followed by changes suggestive of hypoxic ischemic encephalopathy (HIE) 19(18.94\%) cases. Elham et al. [9] has been reported the most frequent MRI finding is brain atrophy in 11(20\%) cases followed by demyelination $10(18.18 \%)$ cases, leukomalecia \& atrophy $7(12.7 \%)$ cases, demyelination \& atrophy $6(10.9 \%)$ cases, basal ganglia lesion $5(9 \%)$ cases, congenital brain malformations in $4(7.3 \%)$ cases, microcephalic changes 3(5.5\%). Aggarwal et al. [2] has been reported hypoxic ischemic encephalopathy as a most common MRI brain finding. 


\title{
V. Conclusions
}

Most children with microcephaly icrocephaly had developmental delay, lower DQ, anaemia, abnormal tone, convulsion, visual abnormality and hearing abnormality. Among the microcephaly children admitted in hospital, severe acute malnutrition was the commonest cause followed by cerebral palsy. Cerebral atrophy was most frequent finding on neuroimaging (MRI) followed by changes suggestive of hypoxic ischemic encephalopathy.

\section{Acknowledgement}

Author acknowledges all the staff from department of pediatrics and staff from the department of radiology for their valuable help in the data collection. Author also acknowledges the help of Mr Sandeep for statistical help.

Contribution: SG: conceptualization, Designed study, Supervised data collection, analaysis and final editing of manuscript.

NK: Data collection, Searching the literature, analysis and manuscript writing.

BS: Data collection, analysis and manuscript writing.

BJ: analysis and manuscript writing.

\section{Conflict of interest: None}

Funding Source: None

\author{
Abbreviations : \\ CBC - Complete Blood Count \\ CHD - Congenital Heart Disease \\ $\mathrm{CP}-$ Cerebral Palsy \\ DQ - Developmental Quotient \\ EEG - Electroencephalography \\ HC - Head Circumference \\ HIE - Hypoxic Ischemic Encephalopathy \\ ICH - Intra Cranial Hemorrhage \\ ITS - Infantile Tremor Syndrome \\ IUGR - Intra Uterine Growth Retardation \\ LFT - Liver Function Test \\ SAM - Severe Acute Malnutrition \\ TBME - Tuberculo Bacterial Meningoencephalitis \\ WHO - World Health Organisation
}

\section{References}

[1]. Agarwal R, Sankhyan N, Jain V. Normal growth and its disorders, In: Paul V K, Bagga A, eds Ghai Essential Pediatrics, Eight edition, New Delhi, CBS Publishers, 2013;7 - 41

[2]. Aggarwal A, Mittal H, Patil R, Debnath S, Rai A. Clinical profile of children with developmental delay and microcephaly. J Neurosci Rural Pract. 2013; 4:288-91.

[3]. Ashwal S, David M, Lauren P, William B. Evaluation of the child with microcephaly (an evidence-based review). Report of the Quality Standards Subcommittee of the American Academy of Neurology and the Practice Committee of the Child Neurology Society, Neurology. 2009; 73: 887-897.

[4]. Barkovich AJ, Ferriero DM, Barr RM. Microlissencephaly a heterogeneous malformation of cortical development. Neuropediatrics. 1999; 29:113-119.

[5]. Baxter PS, Rigby AS, Helene M. Acquired Microcephaly: Causes, Patterns, Motor, IQ Effects and Associated Growth Changes. Pediatrics. 2009; 124:590-595.

[6]. Buros O. Mental Measurements Yearbook. 12th Edition, Lincoln, Buron center for testing, 1995; pp. 263-266.

[7]. Dobyns WB, Andermann E, Andermann F. X-linked malformations of neuronal migration. Neurology. 1996; 47:331-339.

[8]. Doraiswamy PM, Patterson L. Na C, Bicaudate index on magnetic resonance imaging: effects of normal aging. J Geriatr Psychiatry Neurol. 1994; 7(1):13-17.

[9]. Elham A, Nawar, Laila A, et al. Magnetic resonance imaging of the brain in the diagnostic evaluation of microcephaly. Journal of American Science. 2011; 7(3):426- 437.

[10]. Kulkarni ML. Clinical methods in Paediatrics. First edition, New Delhi, Jaypee brother`s medical publisher (P) Ltd, 2005; Vol-III: 163.

[11]. Prasad KM, Girimaji SR, Manjunatha KR, et al. The etiologic patterns in microcephaly with mental retardation. Indian J Psychiatry. $1995 ; 37: 70-80$

[12]. Tatawy SE, Badrawi N, Bishlawy AE. Cerebral Atrophy in Infants with Protein Energy Malnutrition. American Journal Neuroradioligy. 1983;4:434-436

[13]. Van Zagten M, Kessels F, Boiten J, Lodder J. Interobserver agreement in the assessment of cerebral atrophy on CT using bicaudate and sylvian-fissure ratios. Neuroradiology. 1999; 41:261-4.

[14]. Waternberg N, Silver S, Harel S, Lerman-Sagie T. Significance of microcephaly among children with developmental disabilities. J Child Neurol. 2002; 17:117-22.

[15]. Woods C. Human microcephaly. Curr Opin Neurobiol. 2004; 14: 112-117. 